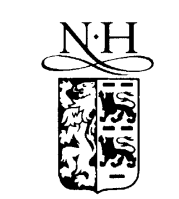

ELSEVIER
Available at

www.ElsevierComputerScience.com

POWERED BY SCIENCE @Direct@

Journal of Systems Architecture 49 (2003) 109-121
JOURNAL OF

SYSTEMS

ARCHITECTURE

www.elsevier.com/locate/sysarc

\title{
Incorporating memory layout in the modeling of message passing programs
}

\author{
F.J. Seinstra *, D. Koelma \\ Intelligent Sensory Information Systems, Faculty of Science, University of Amsterdam, Kruislaan 403, \\ 1098 SJ Amsterdam, The Netherlands
}

\begin{abstract}
One of the most fundamental tasks any automatic parallelization and optimization tool is confronted with is to find an optimal domain decomposition for an application at hand. For regular domain problems (such as simple matrix manipulations) this task may seem trivial. However, communication costs in message passing programs often significantly depend on the capabilities and particular behavior of the applied communication primitives. As a consequence, straightforward domain decompositions may deliver non-optimal performance.

In this paper we introduce a new point-to-point communication model (called P-3PC, or the 'Parameterized model based on the Three Paths of Communication') that is specifically designed to overcome this problem. In comparison with related models (e.g., LogGP) P-3PC is similar in complexity, but more accurate in many situations. Although the model is aimed at MPI's standard point-to-point operations, it is applicable to similar message passing definitions as well.

The effectiveness of the model is tested in a framework for automatic parallelization of image processing applications. Experiments are performed on two Beowulf-type commodity clusters, each having a different interconnection network, and a different MPI implementation. Results show that, where other models frequently fail, P-3PC correctly predicts the communication costs related to any type of domain decomposition.
\end{abstract}

(c) 2003 Elsevier B.V. All rights reserved.

Keywords: MPI; Performance modeling; Automatic domain decomposition

\footnotetext{
Based on "P-3PC: A Point-to-Point Communication Model for Automatic and Optimal Decomposition of Regular Domain Problems" by F.J. Seinstra and D. Koelma, which appeared in IEEE Transactions on Parallel and Distributed Systems 13 (7):758-768, July 2002. (C) 2002 IEEE.

* Corresponding author.

E-mail addresses: fjseins@science.uva.nl (F.J. Seinstra), koelma@science.uva.nl (D. Koelma).
}

\section{Introduction}

For implementing message passing programs MPI [11] has become the de facto standard. Of the many functions defined in MPI, the two blocking point-to-point operations (i.e., MPI_Send ( ) and MPI_Recv ( )) are most important and most often used. To implement optimal message passing applications it is essential to have a performance model that accurately reflects the characteristics of these basic communication primitives. 
In the literature several point-to-point communication models have been described that match the MPI abstractions up to a certain degree (e.g., the Postal Model [4], LogP [6], and LogGP [1]). Although successful in many situations, these models do not incorporate all capabilities of MPI's send and receive operations. Most importantly, the effect of memory layout on communication costs is ignored completely. This is unfortunate, as Prieto et al. [13] have shown that a change in the spatial locality of messages exchanged using MPI can have a severe impact on performance. They state that "the bandwidth reduction due to non-unit-stride memory access could be more significant than the reduction due to contention in the network". Independently, we have come to similar conclusions $[14,15]$. Given these results, it is surprising that no performance model seems to exist that can account for such costs.

In our research, we rely heavily on performance models to perform the task of automatic parallelization of low level image processing applications [16]. As the limitations of existing models proved to be too severe, we have designed a new model that closely matches the behavior of MPI's standard point-to-point operations. The model (called $P-3 P C$, or the 'Parameterized model based on the Three Paths of Communication') bears strong resemblance to the aforementioned models. First, P3PC acknowledges the difference in the time either the sender or the receiver is occupied in a message transfer, and the end-to-end delivery time. Second, P-3PC makes a distinction between communicating data stored either contiguously or non-contiguously in memory. Finally, P-3PC does not assume a strictly linear relationship between the size of a message being transmitted and the communication costs.

This paper is organized as follows. Section 2 discusses the requirements for a model to be applied in our research, and shows the popular LogP and LogGP models not to adhere to these requirements. The P-3PC model is introduced in Section 3. Section 4 shows how P-3PC is applied in the evaluation of communication algorithms executed in a realistic image processing application. In Section 5 model predictions are compared with results obtained on two parallel machines, each having a different interconnection network, and a different MPI implementation. Concluding remarks are given in Section 6.

\section{Model-driven domain decomposition}

The main objective in our research is to build a software architecture that allows image processing researchers to implement parallel applications in a transparent manner [16]. All parallelization issues are to be taken care of by the architecture itself, hidden from the user. The architecture consists of six components (see Fig. 1). The first is an extensive library of data parallel image processing operations. Each operation is annotated with a performance model for run-time cost estimation. One class of models deals with sequential computation only, and another class of models - which is the main topic of this paper-deals with communication. The third component performs a set of

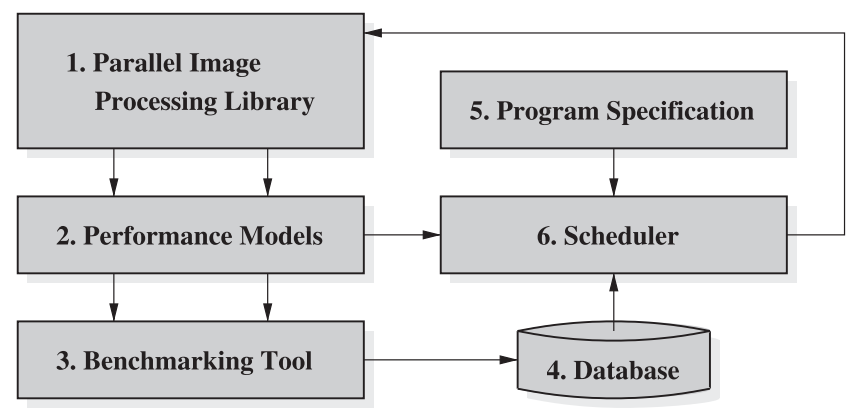

Fig. 1. Simplified architecture overview. 

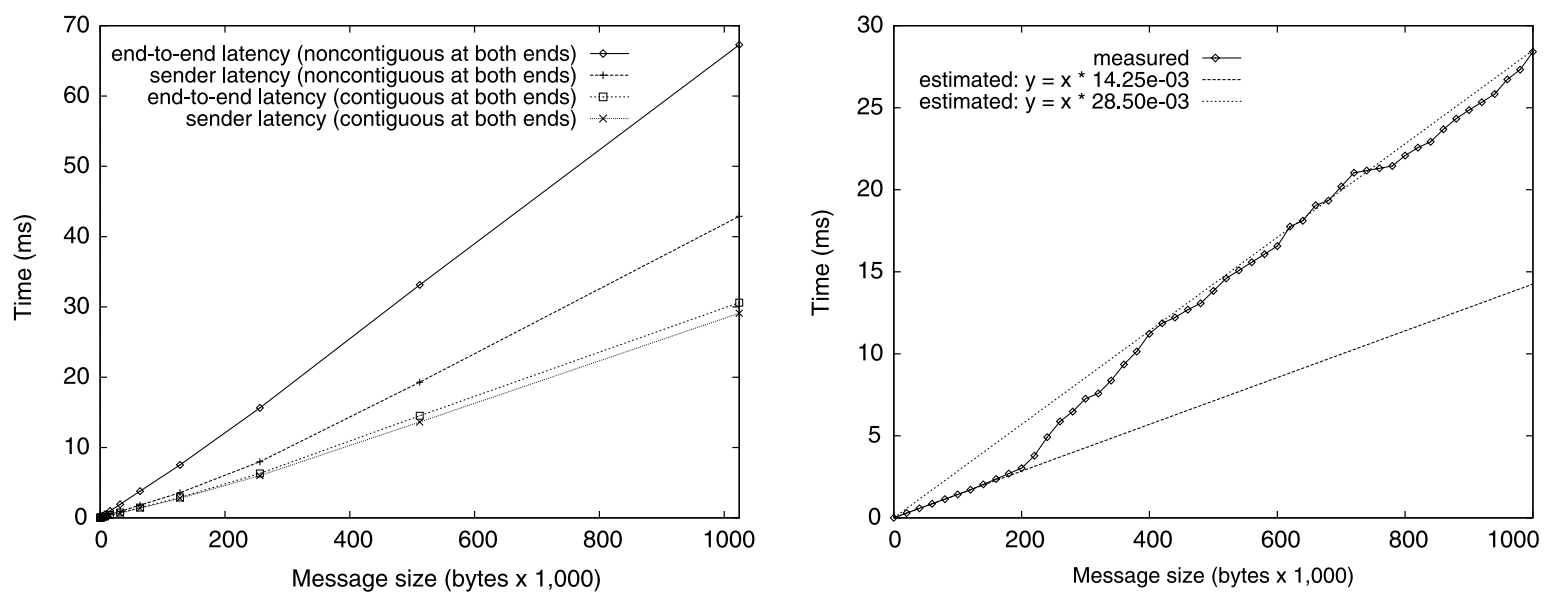

Fig. 2. Values obtained on DAS [3] using MPI-LFC [5] (as described in Section 5): (a) latency: sender side vs. end-to-end and (b) sender latency (detailed).

benchmarking operations to obtain appropriate values for the model parameters. The benchmarking results are stored in a database and used by a scheduler to obtain an optimal schedule for a given application. The scheduling results are routed back to the library to allow each operation to optimize its behavior at run-time.

Based on the models and the measured performance values, it is the task of the scheduler to make optimization decisions regarding: (1) the logical processor grid to map data structures onto (i.e., the actual domain decomposition), (2) the routing pattern for the distribution of data, (3) the number of processing units, and (4) the type of data distribution (e.g., broadcast instead of scatter). In this paper we focus on the first two items as the optimal number of processors and the type of data distribution can be derived from these.

\subsection{Model requirements}

In our library all communication algorithms are implemented using the standard blocking MPI send and receive operations. As low level image processing operations tend to have a bulk synchronous parallel behavior [10,17], usage of any of MPI's additional communication modes is hardly relevant and may even be counterproductive (see [13]). As MPI's collective operations do not provide all functionality required in our library ${ }^{1}$ we have implemented multiple scatter, gather, and broadcast operations in this manner as well.

In data exchange operations the combined latency of sending or receiving multiple messages in sequence may be overlapping with the end-to-end latency of each single message. As shown in Fig. 2(a), such latency differences can be significant. This overlap can be made explicit if a performance model incorporates the following properties:

1. The ability to predict the time a processor is busy executing either the MPI_Send ( ) or the MPI_Recv ( ) operation. As the two communicating nodes may handle the transfer of data differently (see [5], and also requirement 3 in this section), the communication costs at both ends should be modeled independently.

2. The ability to predict the complete end-to-end latency, again independently from the overhead at either node.

\footnotetext{
${ }^{1}$ The main problem with many of the operations defined in MPI 1.1 is that a possibility to define fluctuating strides in multiple dimensions is lacking. Although this problem is lifted in the MPI-2 definition [12] (with the introduction of the MPI_Gatherw ( ) and MPI_Scatterw ( ) operations), as of yet MPI-2 implementations are not generally available.
} 
Depending on the type of domain decomposition, it may be necessary to communicate data stored non-contiguously in memory. Using MPI derived datatypes it is possible to send such data in a single communication step. As was shown by Prieto et al. [13], knowledge of a message's memory layout is important, as non-unit-stride memory access may have a severe impact on performance due to caching. In addition, the MPI operations may even handle the transmission of non-contiguous data differently from contiguous blocks. The MPI 1.1 definition [11] states that "it is up to the implementation to decide whether data should first be packed in a contiguous buffer before being transmitted, or whether it can be collected directly from where it resides". As shown in Fig. 2(a) as well, the latency for communicating either contiguous or non-contiguous data may be significantly different indeed. Such differences can be accounted for if a model incorporates:

3. The ability to reflect the difference in sending data stored contiguously in memory, and noncontiguous data. Again, the memory layout at the two nodes should be modeled independently.

As a consequence from the fact that the send and receive operations are essentially 'black boxes', it is not appropriate to assume communication costs to be linearly dependent on message size. As shown in Fig. 2(b), non-linearities - caused by caching, buffering, packetization, changes in communication policy, etc.-may be quite significant. As a final requirement, a communication model should therefore incorporate.

4. The ability to provide accurate predictions over a large range of message sizes. For the full range of message sizes a strictly linear increase in communication costs should not be assumed.
In certain application areas it may be important to incorporate network contention as well. For our purposes, however, this is not required. In Section 6 we will shortly come back to this issue.

\subsection{Model requirements versus $\log P$ and $\log G P$}

In the literature a multitude of message passing models exists. The two models most relevant for our research are $\log \mathrm{P}[6]$ and $\log G \mathrm{P}$ [1]. $\log \mathrm{P}$ uses four parameters to model the cost of communicating small-sized messages:

- $L$ : the latency associated with message transmission.

- $o$ : the overhead, or the processor occupation time.

- $g$ : the gap, or the minimum time interval between consecutive message transmissions or receptions.

- $P$ : the number of processors.

The LogGP model (see Fig. 3) extends LogP with a linear model for long messages, using one additional parameter:

- $G$ : the gap per byte for long messages.

The two models are important because they make explicit the differences in the occupation time at both ends, and the end-to-end delivery time. Any delay (the gap $g$ ) in consecutive transmissions or receipts is accounted for as well. However, in both models communication overhead is assumed to be identical at both ends. Also, memory layout differences are not incorporated. Finally, the models assume a strictly linear growth rate in the time required for sending messages of arbitrary length. As a consequence, the two models do not

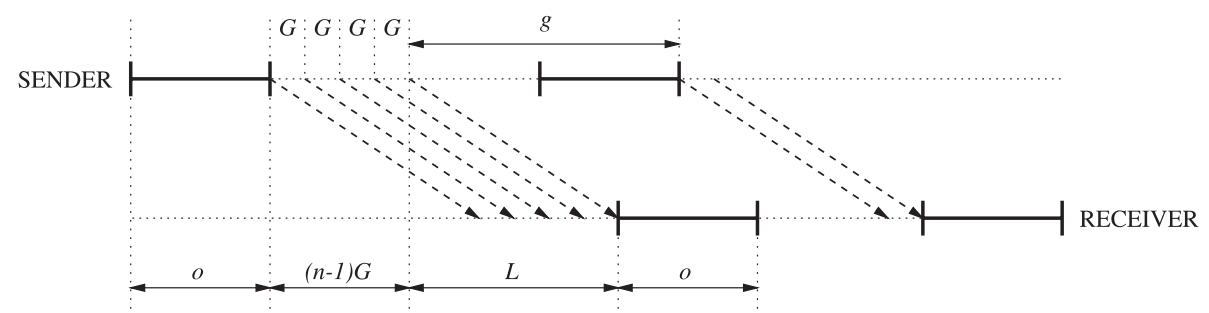

Fig. 3. LogGP communication. 
comply with the specific requirements in our research.

\section{The P-3PC model}

As no model exists that does meet all requirements of Section 2.1, we introduce a new communication model. The model, which we refer to as $P-3 P C$, will be discussed in two parts. First we introduce a simplified version of the complete model (called 3PC) that complies only with the first two requirements of Section 2.1. Subsequently, the $3 \mathrm{PC}$ model is extended to incorporate the remaining two requirements.

\subsection{Part I: $3 P C$}

Given the first two requirements of Section 2.1, we introduce the notion of the three paths of communication, and assume that the cost of message transmission can be captured in three independent values:

- $T_{\text {send }}$ : the cost related to the communication path at the sender (i.e., the time required for executing the MPI_Send ( ) operation).

- $T_{\text {recv }}$ : the cost related to the communication path at the receiver (i.e., the time required for executing the MPI_Recv ( ) operation).

- $T_{\text {full }}$ : the cost related to the full communication path (i.e., the time from the moment the sender initiates a transmission until the receiver has safely stored all data and is ready to continue).
Next, for each path we assume that the communication costs can be represented by two parameters. The transmission of each message is expected to involve a constant amount of time, captured by the mutually independent parameters $t_{\mathrm{cs}}, t_{\mathrm{cr}}$, and $t_{\mathrm{cf}}$ (for the sender, receiver, and full path respectively). For each transmitted byte we assume an 'additional time', which is captured by the mutually independent parameters $t_{\mathrm{as}}, t_{\mathrm{ar}}$, and $t_{\text {af }}$ respectively. The three communication times (see also Fig. 4) involved in the transmission of a message containing $n$ bytes are then given by:

$T_{\text {send }}(n)=t_{\mathrm{cs}}+n \cdot t_{\mathrm{as}}$,

$T_{\text {recv }}(n)=t_{\mathrm{cr}}+n \cdot t_{\mathrm{ar}}$,

$T_{\text {full }}(n)=t_{\mathrm{cf}}+n \cdot t_{\mathrm{af}}$.

Because LogGP's $o$ parameter tends to be equal to $g$ (even for relatively small messages, see [9]), 3PC is identical to LogGP under the following assumptions:

$t_{\mathrm{cs}}=t_{\mathrm{cr}}=g$

$t_{\mathrm{cf}}=2 o+L$,

$t_{\mathrm{as}}=t_{\mathrm{ar}}=t_{\mathrm{af}}=G$.

\subsection{Part II: $P-3 P C$}

To incorporate the last two requirements of Section 2.1, 3PC is 'parameterized' with a cost indicator $\mathbb{M}$, representing the memory layout at the two communicating nodes. Also, it is assumed that each 'additional time' parameter is a function of $n$,

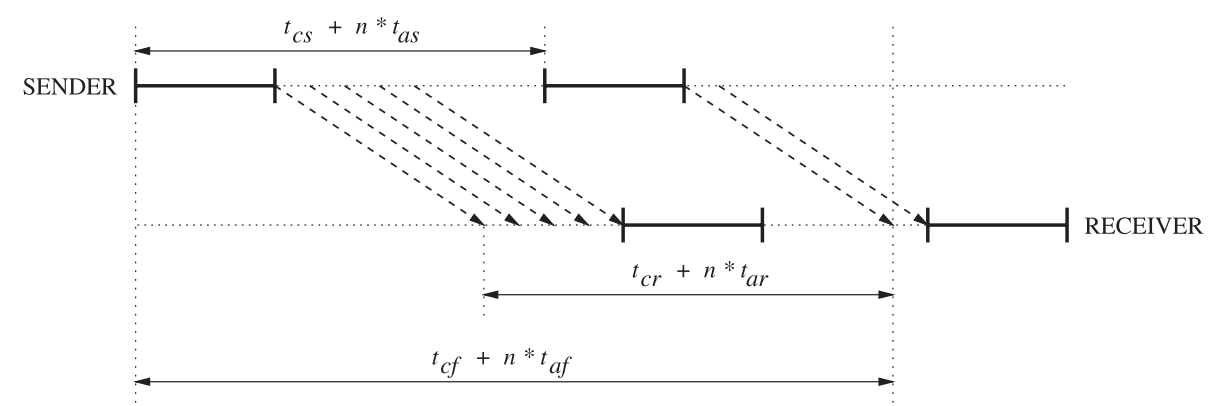

Fig. 4. Communication according to 3PC. 
instead of a constant value for all message sizes. In this extended model (called $P-3 P C$ ), the three communication times involved in a message transfer are given by:

$T_{\mathrm{send}, M}(n)=t_{\mathrm{cs}}+t_{\mathrm{as}, M}(n)$,

$T_{\mathrm{recv}, M}(n)=t_{\mathrm{cr}}+t_{\mathrm{ar}, M}(n)$,

$T_{\mathrm{full}, M}(n)=t_{\mathrm{cf}}+t_{\mathrm{af}, M}(n)$,

where $\mathbb{M} \in\{c c, c n, n c, n n\}$. These layout descriptors indicate the four possible memory layout combinations at the sender and the receiver combined. For example, $c n$ means that a contiguous block of data is transmitted by the sender, which is accepted as a non-contiguous block by the receiver.

As no a priori assumptions can be made about the shape of the 'additional time' functions, a set of benchmarking operations must be performed for several different message sizes. In accordance with the fourth requirement of Section 2.1, each 'additional time' function is then assumed to be piecewise linear between each pair of measured values.

\section{Application of the P-3PC model}

This section shows how the P-3PC model is applied to evaluate the communication costs involved in one of the most essential applications in image processing: i.e., evaluation of the differential structure of images. Examples are edge detection (based on first and second order derivatives) and invariants (based on $i$ th order derivatives).

As is well known, a derivative is best computed using convolution with a separable Gaussian kernel (i.e., $n$ 1-D kernels, each applied in one of the image's $n$ dimensions). The size of the convolution kernel depends on the smoothing scale $\sigma$ and the order of the derivative. In this example (and in the measurements discussed in the next section) we restrict ourselves to first and second order derivatives (five in total) in the $x$ - and $y$-direction of 2-D image data, and $\sigma \in\{1,3,5\}$. Here, for $\sigma=1$, the sizes of the 1-D kernels for the $i$ th order derivative (with $i \in\{0,1,2\}$ ) in any direction are 7,9 , and 9 pixels respectively. For $\sigma=3$ the kernel sizes are
15,23 , and 25 pixels, and for $\sigma=5$ these are 23, 37 , and 39 pixels respectively.

When running such application in parallel, three different communication algorithms are to be executed. First, the input image is to be spread throughout the parallel system in a scatter operation. Second, to obtain partial derivative images pixels in the border regions of each partial input image are to be exchanged among neighboring nodes in the logical CPU grid. Finally, resulting image data is to be gathered at a single node, for on-screen display or storage.

As indicated in Section 2.1, in addition to MPI's collective operations we have implemented multiple versions ourselves, using standard blocking point-to-point operations. Our implementationswhich, in contrast to the MPI versions, allow definition of fluctuating strides in multiple dimensions - are often as fast as the available MPI implementations. This indicates that many MPI distributions are not optimized for a particular machine. Of course, in cases where the MPI implementations are faster, we apply these versions and use the P-3PC estimations for our fastest implementation as an upper bound. In the following, the modeling of such operations is restricted to two implementations, based on either a one-level flat tree (OFT) or a spanning binomial tree (SBT), as shown in Fig. 5.

In case of the OFT scatter operation the root sends out data to all other nodes in sequence. If a $1 \times P$ logical CPU grid is assumed (with $P$ the number of nodes), for each node the data sent out by the root is stored contiguously in memory; for all other grids all data blocks sent out are noncontiguous. Also, for all possible grids all data is accepted as a contiguous block at each receiving node. As each leaf node in the OFT has to wait for all lower-numbered nodes to be serviced by the root before it will receive data itself, the communication costs are highest at either the root node or at the leaf node that is last serviced (depending on the related benchmarking results). A worst case P3PC estimation of this operation is shown in the timeOFTscatter( ) operation in Listing 1. An estimation of the related OFT gather operation is simply obtained by setting $n c$ to $c n$, and changing all occurrences of $T_{\text {send }}$ to $T_{\text {recv }}$. 


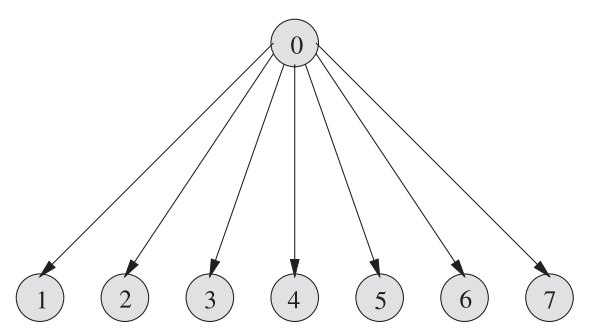

(a)

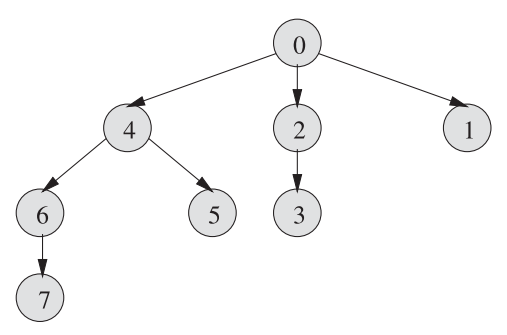

(b)

Fig. 5. Example communication trees: (a) OFT and (b) SBT.

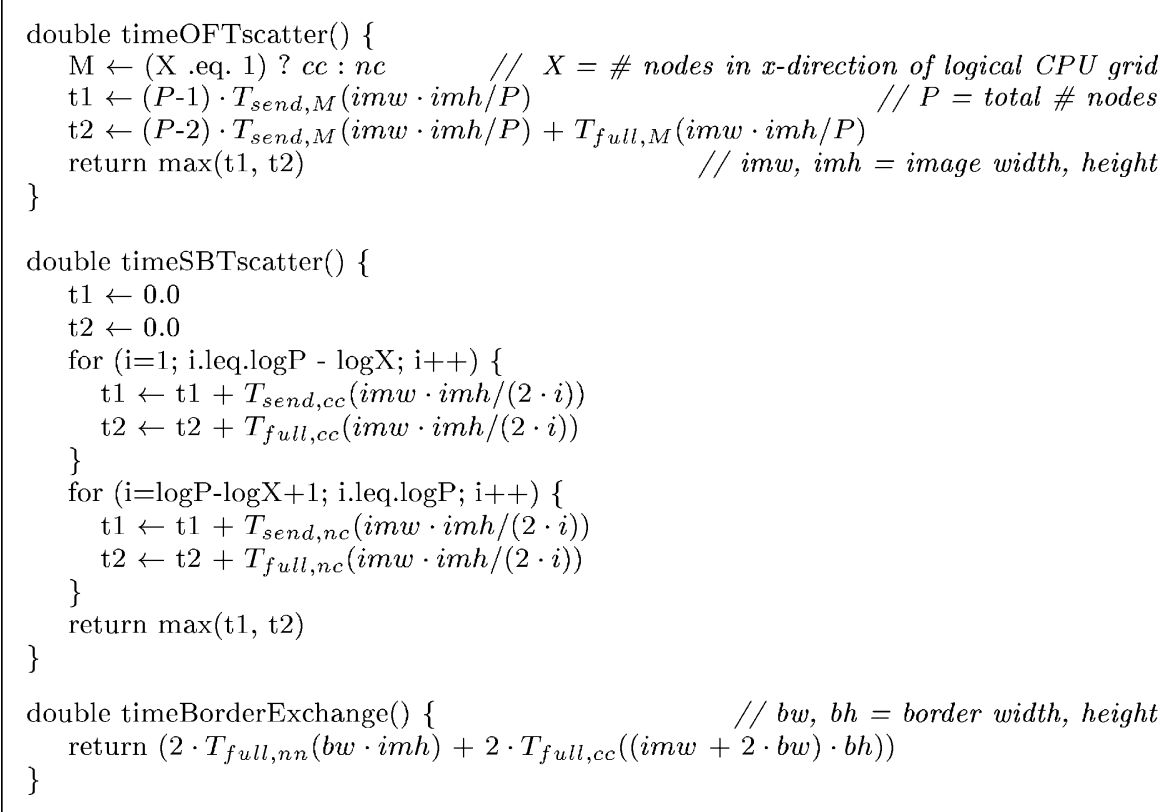

Listing 1. Worst case P-3PC estimations.

In an SBT scatter operation the root transmits data to $\log P$ other nodes. Also, each non-leaf node forwards all received data it is not responsible for. If $X$ is the number of nodes in the $x$ direction of the logical CPU grid, the number of messages involving contiguous data blocks sent out by the root is $\log P-\log X$; the remaining messages sent out are all non-contiguous. In general, the communication costs will be highest at either the root, or the node that is $\log P$ full communication paths away from the root. The timeSBTscatter( ) operation in Listing 1 gives the worst case P-3PC estimation of this op- eration. An estimation of the related SBT gather operation is obtained as before.

A well-known method to implement convolution operations is to extend the domain of the image structure with a scratch border that, on each side of the image in dimension $n$, has a size of about half the 1-D kernel applied in that dimension. When executed in parallel, neighboring nodes in the logical CPU grid need to exchange pixel values to correctly fill the borders of the extended partial images. In our library, the exchange of border data is executed in four steps (see Fig. 6). First, each node sends a subset of its local partial 

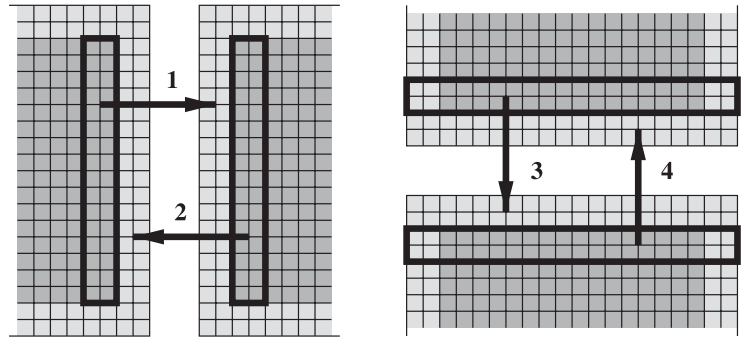

Fig. 6. Border exchange.

image to the neighbor on its right side in the logical CPU grid (if one exists). When a node has accepted this block of data (i.e., after a full communication path period), it subsequently transmits a subset of its local partial image to its neighboring node on the left. As shown in Fig. 6 these steps in the border exchange algorithm always involve non-contiguous blocks of data. Similarly, in the next two steps border data is exchanged in upward and downward direction, in both cases involving contiguous blocks only. Thus, the timeBorderExchange ( ) operation in Listing 1 gives a worst case P-3PC model for this routine.

\section{Measurements and validation}

To validate the P-3PC model we have performed a representative set of benchmarking operations. For each communication path and memory layout combination measurements were performed using four different message sizes, arbitrarily set at $1,50,100$ and $500 \mathrm{~K}$ (all 4-byte values). In addition, benchmarking was performed for 0 -sized messages as well. Note that these values are not chosen to best match the communication characteristics for one particular parallel computer or application. These sizes are representative for messages transmitted in many image processing applications, and are set identically for all machines.

To give full insight in the benchmarking process, Listing 2 gives a simplified overview in pseudocode. To measure communication for noncontiguous data, a fixed number of 100 memory blocks is combined in a single derived datatype definition. For contiguous data only one memory block is used in such definition. As the benchmarking operations are similar to those applied by many others in the literature we leave all further interpretation to the reader.

\subsection{Distributed ASCI supercomputer (DAS)}

The first set of measurements was performed on the 128-node homogeneous DAS-cluster [3] located at the Vrije Universiteit in Amsterdam. All measurements were performed using MPI-LFC [5], an implementation partially optimized for the DAS. The $200 \mathrm{MHz}$ Pentium Pro nodes (with 128 MByte of EDO-RAM) are connected by a 1.2 Gbit/s full-duplex Myrinet network, and run RedHat Linux 6.2.

The performance values obtained for the DAS are presented in Fig. 7. The values indicate that transmitting non-contiguous data indeed has a significant impact on performance. In this case, the additional overhead is due to the fact that MPILFC uses contiguous buffers for non-contiguous data. To preserve the elegance of the benchmarking code, we have measured multiple 'constant time' values for each communication path $(m=0)$. These additional values have no effect on the estimations presented in this section.

In the following we show the results as obtained for the example application of Section 4. In all situations we compare our results with those obtained with LogGP. To avoid using a particularly bad value for the ' $G$ ' parameter, we assume a piecewise linear dependence on message size in the LogGP model as well. Also, to be able to use the measured values of Fig. 7, we have reduced the P3PC model into LogGP as follows: $g=t_{\mathrm{cs}}, L=t_{\mathrm{cf}}$, and $G=t_{\mathrm{af}, c c}$. As indicated in Section 3.1, this reduction makes P-3PC identical to LogGP. Still, to overcome any problem the reader may have with this interpretation of the model, in the remainder we will refer to it as LogGP*.

In Fig. 8(a) results are presented for a $512^{2}$ floating point image, which is mapped onto a $1 \times 16$ logical CPU grid. The graph shows results for the two available implementations of the scatter and gather routines, as well as for the border exchange (for all $\sigma \in\{1,3,5\}$ ). For such 


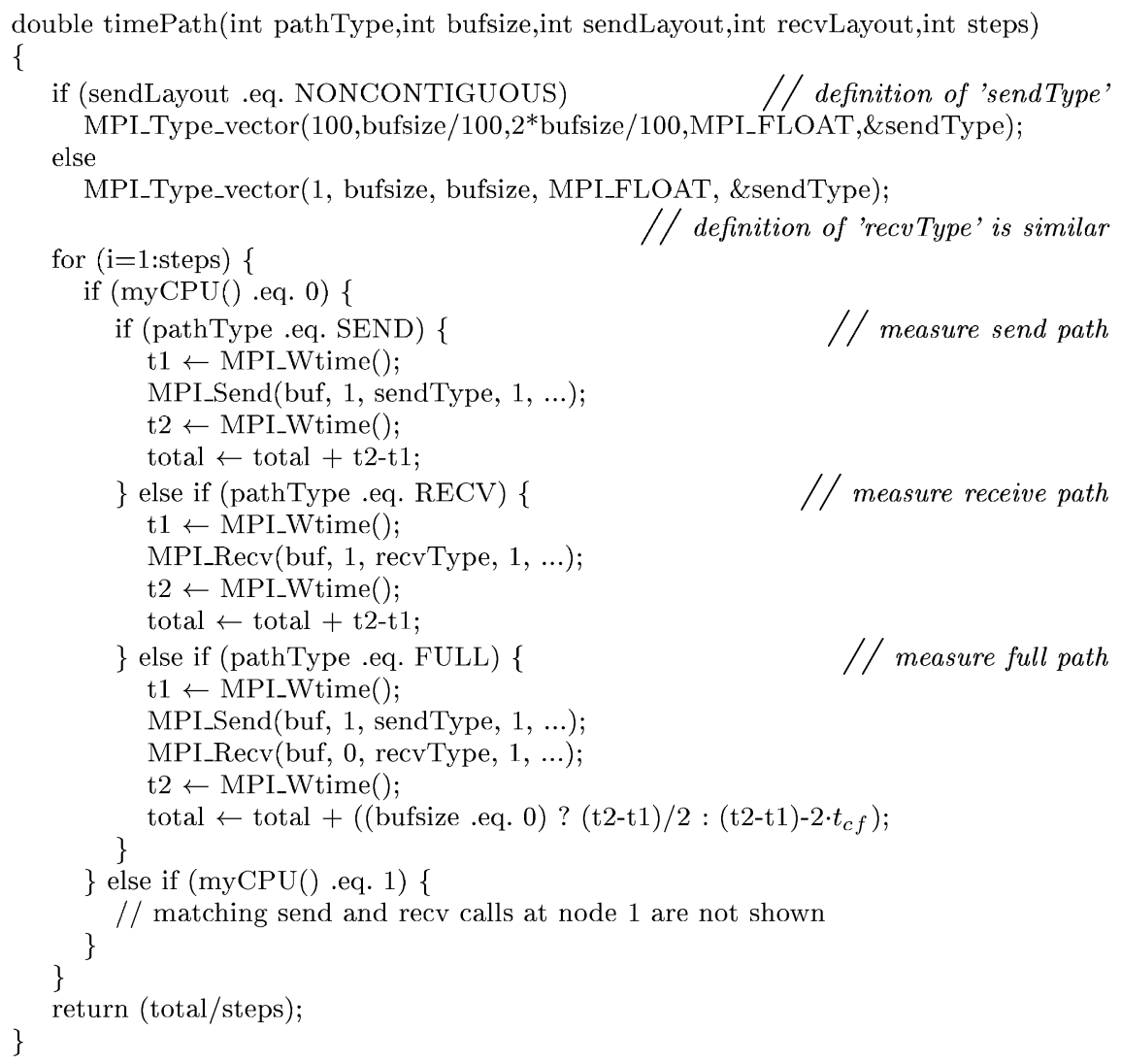

Listing 2. Benchmarking all path-layout combinations.

data decomposition all messages involve contiguous blocks only. The graph shows that P-3PC and LogGP* are both quite accurate for this type of data decomposition. As was to be expected, the estimations obtained from the two models are comparable, although P-3PC seems to do marginally better.

Fig. 8(b) shows that for a $16 \times 1$ data decomposition P-3PC outperforms LogGP* by far. This is because for such decomposition all messages involve non-contiguous data at the sender. Fig. 8 (c) and (d) show similar results for $8 \times 1$ and $32 \times 1$ decompositions. Although most P-3PC estimations are highly accurate, deviations from actual measurements are usually due to small inaccuracies in the performance values obtained by benchmarking. Sometimes, algorithm performance is also slightly degraded by contention in the net- work-an effect not accounted for by P-3PC. However, the impact of memory layout on performance is always more significant than that of contention. Note that this matches the results of [13].

Fig. 8(e) and (f) shows that the P-3PC model indeed allows the scheduler of Section 2 to make correct optimization decisions. According to the LogGP* model, scattering or gathering a $256^{2}$ floating point image is about as expensive for each communication tree and data decomposition. In practice this is not true, however, and P-3PC gives much more accurate estimations at all times.

Fig. 8(g) and (h) shows that P-3PC gives accurate estimates for the border exchange algorithm for all possible data decompositions involving 16 nodes as well. Whereas LogGP* indicates that a $4 \times 4$ decomposition is always optimal (which is 


\begin{tabular}{|l|r|r|r|r|r|}
\hline & $\mathrm{m}=0$ & $\mathrm{~m}=1 \mathrm{~K}$ & $\mathrm{~m}=50 \mathrm{~K}$ & $\mathrm{~m}=100 \mathrm{~K}$ & $\mathrm{~m}=500 \mathrm{~K}$ \\
\hline$T_{\text {send,cc }}(m)$ & 5.98 & 61.72 & 4355.45 & 10246.77 & 58596.98 \\
$T_{\text {send,cn }}(m)$ & 8.04 & 60.74 & 4363.35 & 9853.95 & 57141.29 \\
$T_{\text {send,nc }}(m)$ & 7.93 & 248.88 & 5722.00 & 15142.74 & 90478.81 \\
$T_{\text {send,nn }}(m)$ & 8.29 & 133.88 & 5582.23 & 14137.45 & 87870.27 \\
\hline$T_{\text {recv,cc}}(m)$ & 14.86 & 58.08 & 5754.93 & 12037.78 & 60062.70 \\
$T_{\text {recv,cn}}(m)$ & 14.89 & 127.30 & 9527.59 & 19467.08 & 98016.47 \\
$T_{\text {recv, }, n c}(m)$ & 14.43 & 46.56 & 5517.28 & 12364.45 & 61446.05 \\
$T_{\text {recv, }, n n}(m)$ & 14.82 & 125.05 & 9340.63 & 19685.86 & 98275.11 \\
\hline$T_{f u l l, c c}(m)$ & 23.61 & 131.39 & 4506.32 & 11007.89 & 61277.46 \\
$T_{f u l l, c n}(m)$ & 25.54 & 214.10 & 8665.39 & 19195.53 & 97219.23 \\
$T_{f u l l, n c}(m)$ & 27.05 & 206.94 & 6696.30 & 18015.91 & 95546.60 \\
$T_{f u l l, n n}(m)$ & 24.47 & 287.89 & 11746.29 & 25652.54 & 132399.20 \\
\hline
\end{tabular}

Fig. 7. DAS benchmarking results (in $\mu \mathrm{s}$ ).

explained by the fact that the amount of border data is smallest when each partial image is square), P-3PC correctly prefers the $2 \times 8$ decomposition. Because the exchange of border data may be performed hundreds of times in a realistic application (for example, see [7] for such application that even applies values of $\sigma>5$ ), these results are important indeed.

\subsection{Beowulf at SARA}

The second set of tests was performed on the 40node Beowulf-cluster located at SARA, Amsterdam. On this machine, all measurements and benchmarking were performed using MPICH-1.2.0 [8]. The $700 \mathrm{MHz}$ AMD Athlon nodes (with 256 MByte of RAM) are connected by a $100 \mathrm{Mbit} / \mathrm{s}$ switched Ethernet network, and run Debian Linux 2.2.17.

As the Beowulf cluster is heavily used for other research projects as well, we have been able to use only 8 nodes at a time. Fig. 9 presents results for all algorithms, using a $512^{2}$ floating point image mapped onto a $1 \times 8$ grid as well as a $8 \times 1$ grid. The graphs show that the two models are both quite good in all cases, but P-3PC again provides more accurate estimations. It is clear that the MPICH implementation is much better than the MPI-LFC implementation used on the DAS. Any additional overhead due to non-unit-stride memory access is not caused by buffer copying, but can be attributed to caching alone. Although less significant here, this is exactly the effect Prieto et al. have shown to be important on other parallel machines [13].

\section{Concluding remarks}

In this paper we have presented the new P-3PC model for predicting the communication cost in message passing programs. P-3PC's most important and novel feature is that it models the impact of memory layout on communication costs. In addition, P-3PC incorporates the notion of the 'three paths of communication', and accounts for costs that are not linearly dependent on message size.

P-3PC's predictive power is essential to perform the important task of automatic and optimal decomposition of regular domain problems. Although designed for this specific task, we expect the model to be relevant in other research areas as 


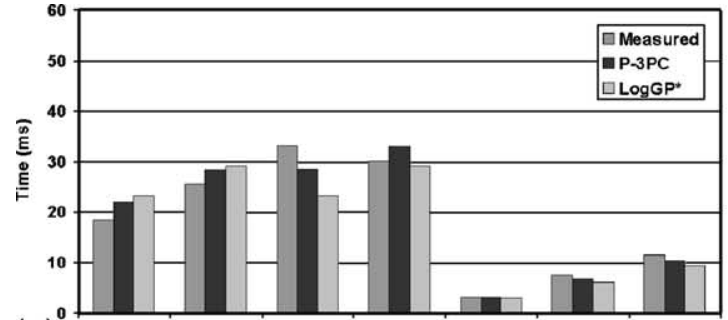

(a) SCAT_OFt SCAT_SBT GATH_OFT GATH_SBT EXCH (1) EXCH (3) EXCH(5)
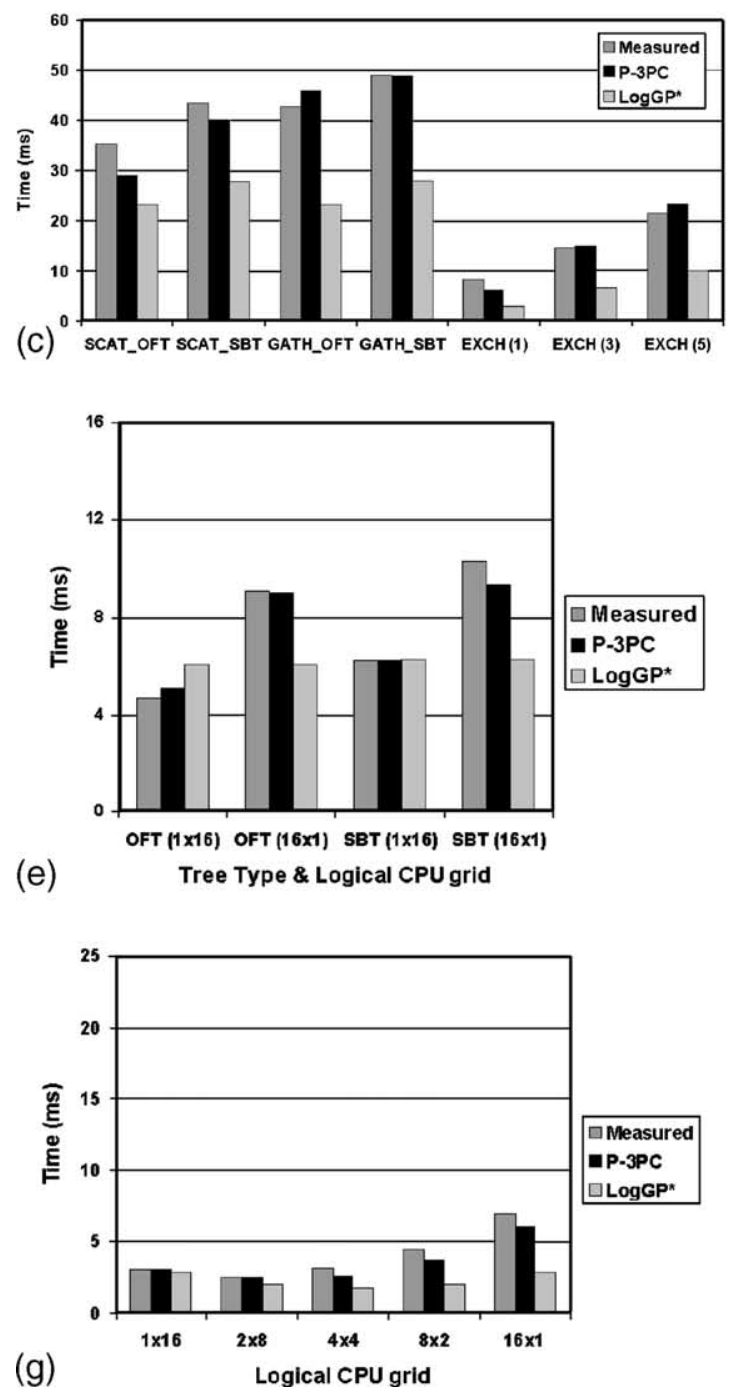

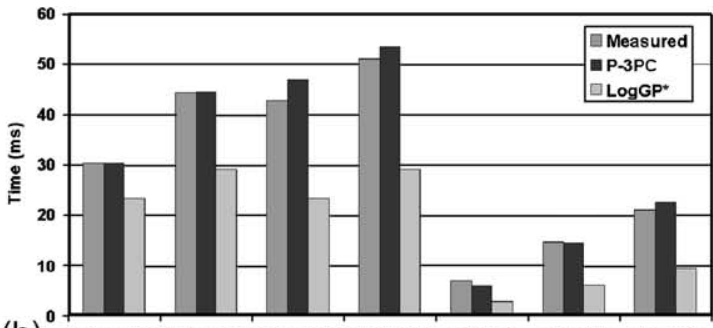

(b) SCAT_OFT SCAT_SBT GATH_OFT GATH_SBT EXCH(1) EXCH (3) EXCH (5)
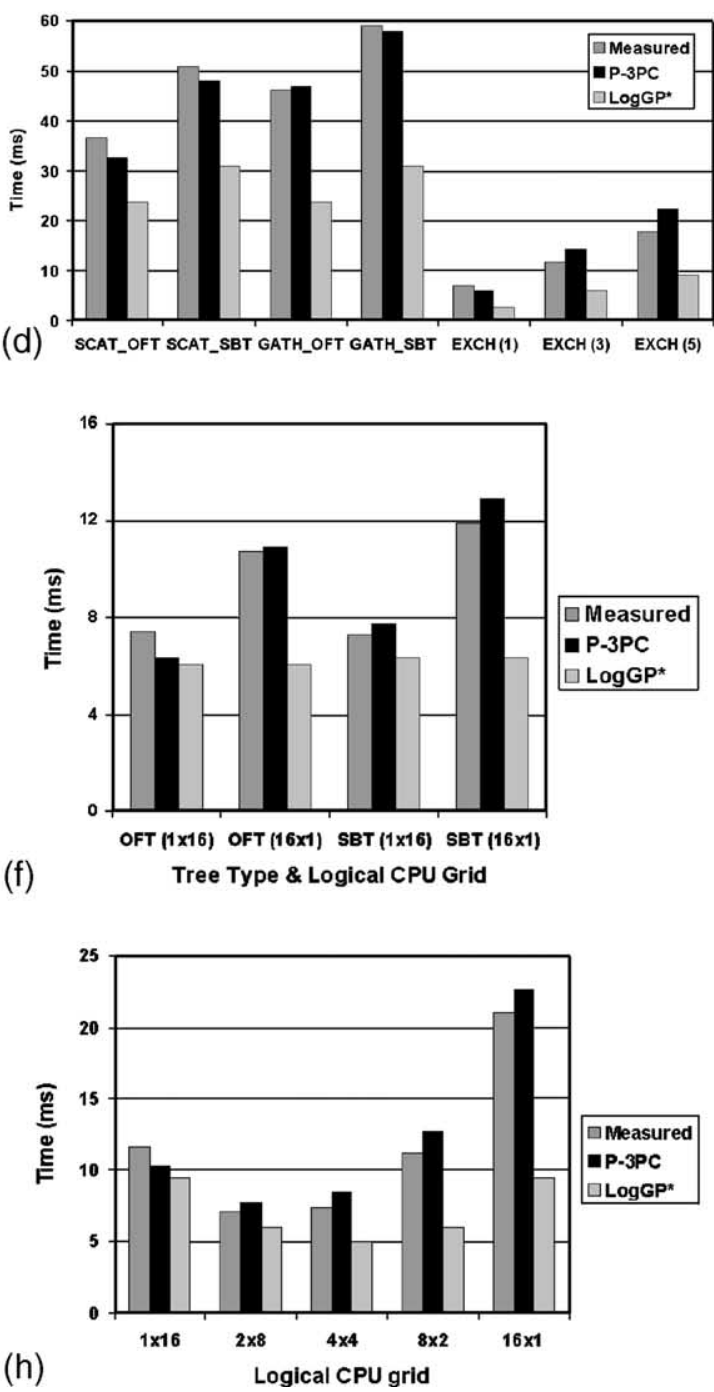

Fig. 8. Measurements (DAS) vs. P-3PC and LogGP* estimations: (a) $512^{2}$ image on $1 \times 16 \mathrm{CPU}$ grid, (b) $512^{2}$ image on $16 \times 1 \mathrm{CPU}$ grid, (c) $512^{2}$ image on $8 \times 1$ CPU grid, (d) $512^{2}$ image on $32 \times 1$ CPU grid, (e) scatter $\left(256^{2}\right.$ image), (f) gather (256 image), (g) exchange $\left(512^{2}\right.$ image, $\left.\sigma=1\right)$ and $(\mathrm{h})$ exchange $\left(512^{2}\right.$ image, $\left.\sigma=5\right)$. 

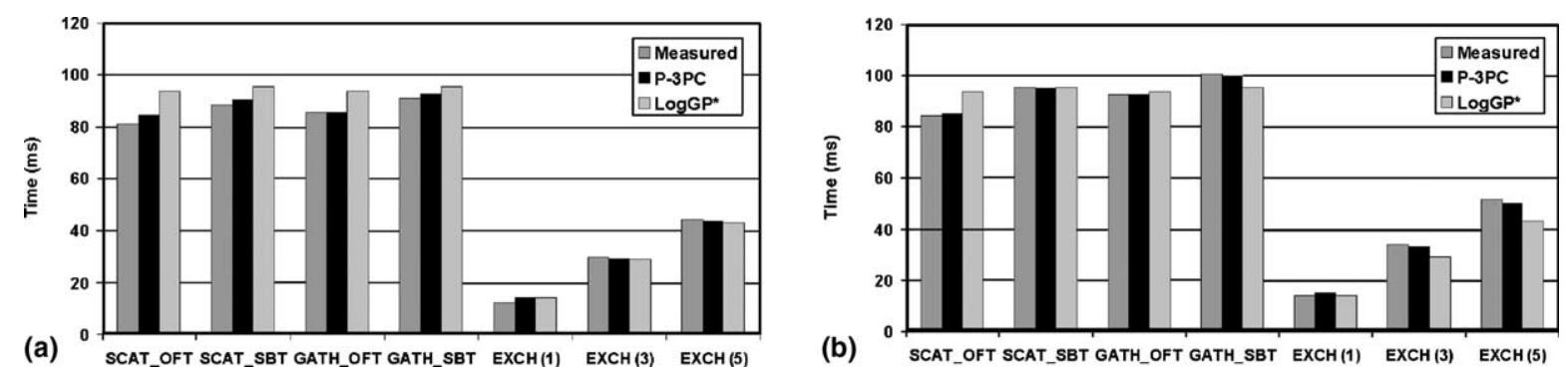

Fig. 9. Measurements (Beowulf) vs. P-3PC and LogGP* estimations: (a) $512^{2}$ image on $1 \times 8$ CPU grid and (b) $512^{2}$ image on $8 \times 1$ CPU grid.

well. It is important to note, however, that P-3PC suffers from the same problem as other models that abstract from the actual network topology (see also [6]). The model cannot discriminate between algorithms that cause severe network contention, and those that do not. In our research this is not a problem, as we only apply communication patterns that are expected to perform well on most network topologies used today. Still, because P3PC is similar to the LogGP model, it can easily be extended to account for contention, in the same manner as described in [2].

As the P-3PC model stresses the importance of benchmarking to obtain accurate values for the model parameters, one may argue that the predictive power of the model is limited. However, the model does not specifically enforce a large number of measurements to be performed. As for models that incorporate a similar level of abstraction, a set of two or three measurements for each communication path may already be sufficient to obtain accurate predictions. The P-3PC model merely acknowledges that non-linearities in communication costs may be significant (as shown in Section 2.1) and should be accounted for.

We are aware of the fact that an evaluation of $\mathrm{P}-3 \mathrm{PC}$ is never complete. However, the evaluation as presented here-incorporating two networks and two MPI versions - has shown the model to be highly accurate in estimating the communication costs related to any type of domain decomposition used in a realistic imaging application. As such, we have shown P-3PC to be useful as a basis for automatic and optimal decomposition of regular domain problems.

\section{References}

[1] A. Alexandrov, M. Ionescu, K. Schauser, C. Scheiman, LogGP: incorporating long messages into the $\log P$ model-one step closer towards a realistic model for parallel computation, in: Proceedings of the Symposium on Parallel Algorithms and Architectures (SPAA), Santa Barbara, California, USA, July 1995, pp. 95-105.

[2] C. Andras Moritz, M. Frank, LoGPC: modeling network contention in message-passing programs, IEEE Transactions on Parallel and Distributed Systems 12 (4) (2001) 404-415.

[3] H. Bal et al., The distributed ASCI supercomputer project, Operating Systems Review 34 (4) (2000) 76-96.

[4] A. Bar-Noy, S. Kipnis, Designing broadcasting algorithms in the postal model for message-passing systems, Mathematical Systems Theory 27 (5) (1994) 431-452.

[5] R. Bhoedjang, T. Rühl, H. Bal, LFC: a communication substrate for Myrinet, in: ASCI'98, Proceedings of the Fourth Annual Conference of the Advanced School for Computing and Imaging, Lommel, Belgium, June 1998, pp. 31-37.

[6] D. Culler et al., LogP: towards a realistic model of parallel computation, in: Proceedings of the Fourth ACM SIGPLAN Symposium on Principles and Practice of Parallel Programming, San Diego, California, May 1993, pp. 1-12.

[7] J. Geusebroek, A. Smeulders, H. Geerts, A minimum cost approach for segmenting networks of lines, International Journal of Computer Vision 43 (2) (2001) 99-111.

[8] W. Gropp, E. Lusk, N. Doss, A. Skjellum, A highperformance, portable implementation of the MPI message passing interface standard, Parallel Computing 22 (6) (1996) 789-828.

[9] M. Lauria, LogP characterization of FM on the VU's DAS machine, Technical Report, Dipartimento di Informatica e Sistemistica, Universita di Napoli Federico II, 1997.

[10] W. McColl, Scalability, portability and predictability: the BSP approach to parallel programming, Future Generation Computer Systems 12 (1996) 265-272.

[11] Message Passing Interface Forum, MPI: a message-passing interface standard (version 1.1), Technical Report, Uni- 
versity of Tennessee, Knoxville, Tennessee, June 1995. Available from http://www.mpi-forum.org/.

[12] Message Passing Interface Forum, MPI-2: extensions to the message-passing interface, Technical Report, University of Tennessee, Knoxville, Tennessee, July 1997. Available from http://www.mpi-forum.org/.

[13] M. Prieto, I. Llorente, F. Tirado, Data locality exploitation in the decomposition of regular domain problems, IEEE Transactions on Parallel and Distributed Systems 11 (11) (2000) 1141-1149.

[14] F. Seinstra, D. Koelma, Modeling performance of low level image processing routines on MIMD computers, in: ASCI'99, Proceedings of the Fifth Annual Conference of the Advanced School for Computing and Imaging, Heijen, The Netherlands, June 1999, pp. 307-314.

[15] F. Seinstra, D. Koelma, P-3PC: a point-to-point communication model for automatic and optimal decomposition of regular domain problems, IEEE Transactions on Parallel and Distributed Systems 13 (7) (2002) 758-768.

[16] F. Seinstra, D. Koelma, J. Geusebroek, A software architecture for user transparent parallel image processing, Parallel Computing 28 (7-8) (2001) 967-993.

[17] L. Valiant, A bridging model for parallel computation, Communications of the ACM 33 (8) (1990) 103-111.

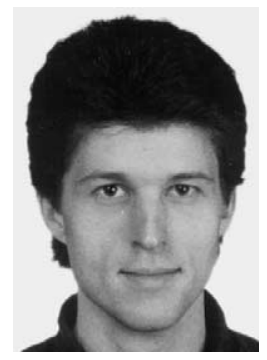

Frank Seinstra received the M.Sc. degree in computer science from the Vrije Universiteit in Amsterdam in 1996. He is currently finishing his Ph.D. thesis (entitled: 'User Transparent Parallel Image Processing') at the University of Amsterdam. His research interests include parallel and distributed programming, automatic parallelization, performance modeling, and scheduling - especially in the application area of image and video processing.

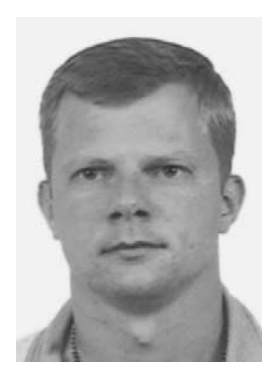

Dennis Koelma received the M.Sc. and $\mathrm{Ph} . \mathrm{D}$. degrees in computer science from the University of Amsterdam in 1989 and 1996, respectively. The subject of his thesis is "A Software Environment for Image Interpretation". $\mathrm{He}$ is currently working on Horus: a software architecture for research in digital image processing. His research interests include image and video processing, software architectures, parallel programming, databases, graphical user interfaces, and image information systems. 